\title{
Selection of a geographic area of interest for syndromic surveillance
}

\section{Erica FOUGERE, Delphine CASAMATTA, Guillaume SPACCAFERRI}

Santé publique France, Lyon, Auvergne-Rhône-Alpes, France

Objective

Define analytic areas at a sub-regional level to better meet the needs of local decision-makers.

\section{Introduction}

Since 1 January 2016, the Auvergne and Rhône-Alpes regions have merged as part of the territorial reform. The new region is composed of 12 departments and accounts for more than 8 million inhabitants. Its territory is heterogeneous in population density with very urban areas (Clermont-Ferrand, Grenoble, Lyon and Saint-Etienne) and important mountainous areas (Arc Alpin, Massif Central). In France since 2004, the syndromic surveillance system SurSaUD® [1] coordinated by the French Public Health Agency (Santé publique France) collects morbidity data on a daily basis from two data sources: the emergency departments (ED) network Oscour ${ }^{\circledR}$ and the emergency general practitioners SOS Médecins associations. In Auvergne-Rhône-Alpes, the number of structures participating in the scheme has gradually increased from 2006 to today; as of 1 September 2018, all emergency services $(\mathrm{N}=84)$ and all SOS Médecins associations $(\mathrm{N}=7)$ transmit their data on a daily basis. Both data sources collect medical diagnoses, using ICD10 codes in the ED network and specific medical thesaurus in SOS Médecins Associations.

These data are routinely analyzed to detect and follow-up various expected or unusual public health events all over the territory [2]. A reflection on the analysis of monitoring data at the sub-regional level was conducted in the region in order to refine the analyses carried out and better meet the expectations of local partners.

\section{Method}

The SurSaUD® system has been progressively upgraded in recent years reaching its regional completeness in 2018. At the same time, the quality of the data also improved, making it possible to work on finer spatial levels.

Three infra-regional partitioning scenarios were studied: the Territorial Hospital Groups (GHT) created in 2016. Although they represents 15 groupings in our region, the 12 departments and the 3 emergency physicians networks, we wondered about the possibility of carrying out analyzes on this scale to answer to local needs. The former Rhône-Alpes region had the particularity of being endowed with three networks of emergency physicians rooted in the region for more than 10 years. These networks are:

- The RENAU network (North Alpine Emergency Network) which covers the Savoie, Haute-Savoie, and part of Isère regions (including Grenoble University Hospital);

- The RESUVAL network (emergency network of the Rhone Valley) which covers the Ain, the Rhône (including the University Hospital of Lyon), the Drôme, the Ardèche (except the northern part) and the rest of the Isère regions;

- The REULIAN network (Loire and northern Ardèche emergency network) which covers the Loire (including the University Hospital of Saint-Etienne) and the northern part of Ardèche (Annonay).

These networks were created to structure the sectors and the organization of health care provision in emergency medicine.

Results

A breakdown into departments seemed of little relevance due to the large number of departments within the region and the significant heterogeneity in terms of activity, data transmission and quality. At this departmental scale, the volume of activity in the number of emergency reports transmitted varies from 51,300 to 608,400 annual visits depending on the department. Similarly, the coding of diagnoses varies between $19 \%$ and $81 \%$, depending on the department. 
For the 15 GHTs, very recent in the region, the organization is not yet homogeneous in terms of healthcare provision and business software. Indeed, GHTs represent between 53,000 and 514,000 annual emergency visits. In addition, the quality of the data provided varies from 10 to $94 \%$ of coded diagnoses. This breakdown was thus not finally retained.

In order to have coherent territories of relatively homogeneous weight, three sectors were defined: the first two sectors relied on the networks of emergency physicians in the east and center of the region (RENAU and RésuVal). For the west, the territory of the REULIAN network was merged with the former Auvergne region, which was also consistent with medical practices. Consequently, these three networks make it possible to have a logic of organization of health care and present relatively balanced sectors. These sectors comprise between 21 and 34 emergency services, and account for one to three SOS Médecins associations. This represents between 545,000 and 1,028,000 annual visits to emergency services, and between 93,000 and 109,000 annual consultations with SOS Médecins. The transmission quality of emergency visits data at D 1 varies between $51 \%$ and $73 \%$. A breakdown into 3 sectors (West, Center, and East) was finally retained. To illustrate the situation, a study on the characteristics and sub-regional spatio-temporal diffusion of respiratory syncytial virus (RSV) bronchiolitis epidemics in children under 2 years in the AuvergneRhône-Alpes region did not show any major differences between sectors to identify the start of the outbreak (+/- 1 or 2 weeks). However, the identification of the start of the outbreak in 1 or 2 sectors only did not seem to trigger outbreak onset at regional level. There is therefore a delay in informing the start of the outbreak for the sector (and the facilities) concerned, which may also delay the possibility to adapt health care provision with respect to the epidemic peak. Another use of these sectors was performed during the surveillance of allergies in the region. In the spring, the pollens are different from one week to another and depending on the territory. Also, thanks to these three sectors, it is possible to refine the observations to allow a prevention closer to the field. In the spring of 2018, in the East sector, a peak higher than in previous years was observed, while in the other two sectors, this dynamics was relatively similar to the one observed over the last two years.

\section{Conclusions}

The 3 networks of emergency physicians in the former Rhône-Alpes region, which have been active for more than 10 years, show that there is a real logic of health care provision between facilities belonging to the same network (common health problems, geographical characteristics, etc.). It was therefore relevant to rely on these networks to propose a sub-regional breakdown. In order to balance the sectors (in terms of the number of reports of emergency visits, number of facilities, population), it was decided to group within the Western sector the REULIAN territory and the territory of the former Auvergne

The results of the study on bronchiolitis show that the analysis of the spread of RSV-related bronchiolitis outbreaks at the subregional level could allow a better anticipation of outbreak onset, and therefore of the epidemic peak which constitutes the main stake for the organization of health care provision. The interest of this surveillance in sectors will also have to be evaluated for other seasonal disease.

\section{Acknowledgement}

To OSCOUR $®$ and SOS Médecins regional partners and to the Intervention Unit of Santé publique France in Auvergne-RhôneAlpes.

\section{References}

1. Caserio-Schönemann C, Bousquet V, Fouillet A, Henry V. 2014. Le système de surveillance syndromique SurSaUD (R). Bull Epidemiol Hebd (Paris). (3-4), 38-44.

2. Josseran L, Nicolau J, Caillère N, Astagneau P, Brücker G. 2006. Syndromic surveillance based on emergency department activity and crude mortality: two examples. Euro Surveill. 11(12), 225-29. PubMed https://doi.org/10.2807/esm.11.12.00668-en 


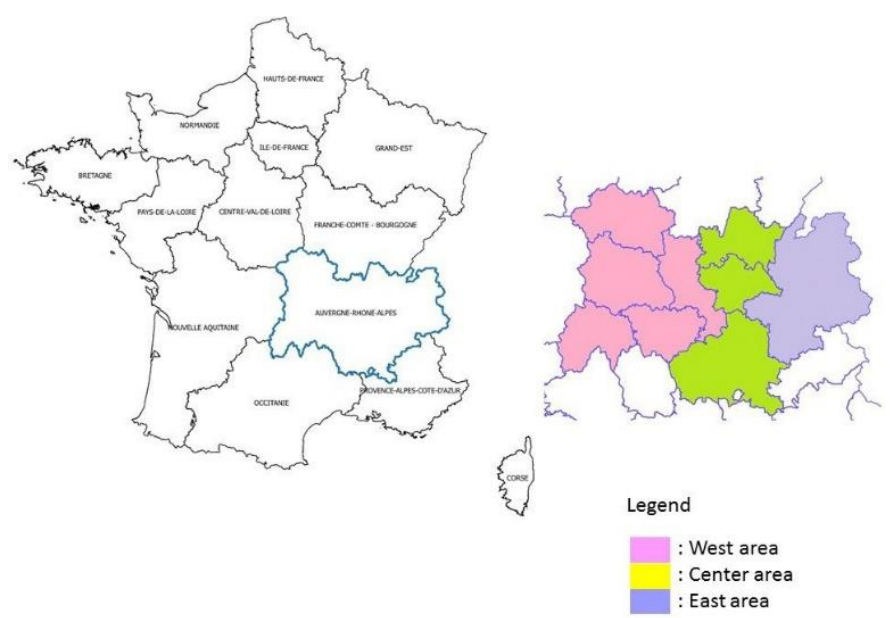

Figure 1. Regional map of metropolitan France (left) and geographic area of interest for syndromic surveillance in ARA (right) 